\title{
AVALIAÇÃO DA VISCOSIDADE APARENTE DE PASTAS DE AMIDOS NOS VISCOSÍMETROS BROOKFIELD RVDV-II+ PRO E RÁPIDO VISCO- ANALISADOR RVA-4
}

\section{EVALUATION OF APPARENT VISCOSITY OF STARCH PASTES IN BROOKFIELD RVDV-II + PRO AND RAPID VISCO ANALYZER RVA-4 VISCOMETERS}

\author{
Manoela Estefânea Boff Zortéa ${ }^{1}$; Ivo Mottin Demiate ${ }^{1}$; Marco Aurélio Praxedes ${ }^{1}$; Gilvan Wosiacki ${ }^{1}$ \\ ${ }^{1}$ Universidade Estadual de Ponta Grossa - UEPG - Ponta Grossa - Brasil. \\ E-mail: demiate@yahoo.com
}

\begin{abstract}
Resumo
Viscosímetros rotacionais são instrumentos utilizados para avaliar a viscosidade em diferentes tipos de produtos, incluindo suspensões e soluções de polissacarídeos como o amido, com destaque para usos na indústria alimentícia. Neste trabalho foi feita a comparação entre dois equipamentos, o Brookfield RVDV-II+PRO e o Rápido Visco-Analisador RVA-4, usando-se as mesmas condições de análises e teve por objetivo específico permitir a comparação direta entre as medidas obtidas nos dois sistemas para facilitar a utilização dos equipamentos principalmente pela indústria. Pastas de amido foram produzidas em água deionizada e submetidas a análises sob as mesmas condições nos diferentes viscosímetros. Os resultados foram obtidos da relação que foi feita em gráfico composto pela viscosidade aparente $(\mathrm{cP})$ e torque (\%), partindo-se de no mínimo 18 pontos selecionados pelas temperaturas em comum. $O$ que se observou pelas análises desenvolvidas com os amidos nativos de mandioca, milho e mandioquinha-salsa, amidos de mandioca ácidomodificado 0,15 mol $\mathrm{L}^{-1}$ ( $\mathrm{HCl}$ e oxidado 2,0 \% ( $\mathrm{NaClO}$ ) e polvilho azedo, foi que, para a mesma amostra, os valores de torque em um viscosímetro e de viscosidade em outro, estão diretamente relacionados, obtendo-se valores de $R^{2}$ próximos de 1 , o que comprovou a possibilidade de uma interconversão entre os valores dos dois equipamentos através de equações para cada comportamento.
\end{abstract}

Palavras-chave: viscosímetro; RVA-4; viscosidade aparente; amido.

\section{Introdução}

O amido é o produto final do processo fotossintético e constitui a principal reserva de carbono das plantas. Sua formação ocorre devido à atividade coordenada de algumas enzimas, tanto nas organelas fotossinteticamente ativas, onde o amido é reserva temporária, quanto nos amiloplastos de órgãos de reserva (CEREDA, 2001).

O amido isolado dos vegetais é composto essencialmente por polímeros de amilose, 
polissacarídeo linear de $(1 \rightarrow 4)$ - $\alpha$-D-glucose e amilopectina, polissacarídeo ramificado, onde cadeias de $(1 \rightarrow 4)$ - $\alpha$-D-glucana são conectadas por ligações $\alpha(1 \rightarrow 6)$. O amido deve muito de sua funcionalidade a estas duas macromoléculas, assim como a organização física das mesmas dentro da estrutura granular, sendo que os teores de amilose e amilopectina variam com a espécie botânica (BILIADERIS, 1991).

Devido ao relativo baixo custo, o amido tem sido muito utilizado pela indústria alimentícia como ingrediente calórico e como melhorador de propriedades físico-químicas. É utilizado para alterar ou controlar diversas características como textura, aparência, umidade, consistência e estabilidade no armazenamento (shelf-life). Pode também ser usado para ligar ou desintegrar; expandir ou adensar; clarear ou tornar opaco; reter a umidade ou inibi-la; produzir textura lisa ou polposa e coberturas leves ou crocantes. Também serve tanto para estabilizar emulsões quanto para formar filmes resistentes ao óleo (CEREDA, 2001). O mercado de amido vem crescendo e se aperfeiçoando continuamente, levando à busca de produtos com características específicas que atendam as exigências industriais (CEREDA e WOSIACKI, 1985; VILPOUX, 1998).

As propriedades tecnológicas de utilização dos amidos envolvem suas características físicas, químicas e funcionais. A aplicação do amido para determinada finalidade em processo industrial depende de suas propriedades funcionais como temperatura de gelatinização, poder de inchamento (absorção de água), viscosidade, retrogradação e expansão (CEREDA e VILPOUX, 2003).

A viscosidade é uma das medidas utilizadas para a caracterização reológica de diferentes tipos de produtos. De maneira geral, entende-se por propriedade reológica aquela que especifica a deformação ou a taxa de deformação que uma substância apresenta quando sujeita a uma tensão. A viscosidade de um líquido mede a resistência interna oferecida ao movimento relativo das diferentes partes desse líquido, ou seja, a resistência ao fluxo (VILLETTI, 2003).

Suspensões de amido em água, ao serem submetidas a aquecimento, passam por um repentino aumento de viscosidade, que pode ser registrado por intermédio de viscosímetros rotacionais quando os grânulos começam a inchar. Neste ponto, polímeros com menor peso molecular, particularmente moléculas de amilose, começam a ser lixiviadas dos grânulos. Um pico de viscosidade é obtido durante o empastamento, quando os grânulos, em sua maioria, estão totalmente inchados, havendo também grânulos intactos e o alinhamento molecular dos polímeros solubilizados ainda não ocorreu dentro do campo de atrito do instrumento. Caso haja manutenção da temperatura a $95^{\circ} \mathrm{C}$ e agitação constante, os grânulos começam a se romper, a solubilização dos polímeros continua e o alinhamento molecular ocorre dentro do campo de atrito do instrumento, causando uma diminuição da viscosidade (THOMAS e ATWELL, 1999).

Ao ocorrer resfriamento, alguns polissacarídeos liberados do interior dos grânulos começam a se reassociar, formando um precipitado ou gel ocorrendo aumento na opacidade da pasta. Este 
processo é chamado retrogradação ou setback (WHISTLER e BEMILLER, 1997) e ocorre aumento da viscosidade. Dependendo do tipo de amido (da fonte botânica, ou se é um amido natural ou modificado), do nível de sólidos solúveis, do $\mathrm{pH}$ e do regime de aquecimento, vários perfis de gelatinização e empastamento podem ser gerados (THOMAS e ATWELL, 1999) e observados em instrumentos utilizados para medir essa viscosidade.

As mudanças de viscosidade em suspensões amiláceas, devido ao intumescimento do grânulo de amido durante o aquecimento são comumente avaliadas em viscosímetros rotacionais especiais denominados de viscoamilógrafos, sendo os mais amplamente utilizados o da marca Brabender (Duisburg, Alemanha) e o Rápido Viscoanalisador (RVA), fabricado pela Newport Scientific (Warriewood, Austrália). Deffenbaugh \& Walker (1989) compararam esses dois viscoamilógrafos para a determinação de propriedades de empastamento de amidos. De acordo com os autores, o viscoamilógrafo Brabender, desde o início de sua comercialização em 1930, se tornou um equipamento chave para a indústria processadora de amidos e de produtos amiláceos. Como limitações do referido equipamento, os autores citam a necessidade de grande quantidade de amostra (geralmente entre 30,0 e 60,0 g) e o tempo de análise que comumente supera 90 minutos.

A unidade de pesquisa de trigo da Agência Nacional Australiana de Ciência (CSIRO) e o Bread Research Institute (Austrália) desenvolveram, em conjunto, o Rápido Viscoanalisador (RVA) com o principal propósito de estimar rapidamente danos à qualidade do trigo devido ao brotamento (ROSS et al., 1987). Para essa aplicação, o RVA necessitava de apenas $4 \mathrm{~g}$ de amostra, era robusto e simples de operar, produzindo os resultados em apenas três minutos. Para o estudo do empastamento de amidos, os primeiros relatos científicos do uso do RVA são dos anos 1988 e 1989 e as vantagens são a pequena quantidade de amostra (geralmente entre 1,8 a 3,0 g) e o reduzido tempo de análise (geralmente entre 13 e 25 minutos), além da durabilidade, simplicidade de operação e da possibilidade de comparação dos valores obtidos com aqueles do viscoamilógrafo Brabender (DEFFENBAUGH e WALKER, 1989).

A avaliação da viscosidade de amidos feita no aparelho Brabender apresenta uma boa habilidade discriminativa no perfil de empastamento. Entretanto, o longo tempo de análise, a grande quantidade de amostra requerida, a pequena reprodutibilidade de instrumento para instrumento e o difícil procedimento de calibração vêm motivando o uso do RVA, que está se tornando muito popular para a análise das propriedades de pasta dos amidos (THOMAS e ATWELL, 1999).

O Rápido Viscoanalisador modelo RVA-4 é um viscosímetro rotacional muito utilizado para a avaliação da qualidade de amidos e farinhas em muitos países (THOMAS; ATWELL, 1999). O perfil de empastamento de amidos obtidos pelo RVA inclui pico de viscosidade, tempo para atingir esse pico, quebra, viscosidade final e temperatura de pasta.

Outro viscosímetro rotacional existente, da Brookfield Engineering Laboratories 
(Middleboro, MA, EUA), é, sem dúvida, um dos instrumentos mais utilizados mundialmente na medida de viscosidade de diversos tipos de preparações fluidas. Devido a seu custo modesto em relação a outros viscosímetros de velocidade variável, encontra grande aplicação nos laboratórios de controle de qualidade das indústrias químicas, alimentícias de cosméticos, entre outras (SILVEIRA, 1991).

Em resposta a uma demanda da indústria americana de moagem úmida de milho por novos equipamentos para avaliação do comportamento reológico de suspensões de amido quando submetidos ao aquecimento, Steffe et al. (1989) desenvolveram um projeto de um viscosímetro rotacional Brookfield dotado de uma célula para pequenas amostras, um spindle tipo flag impeller (que possibilita com a rotação manter os grânulos de amido em suspensão durante a análise) e conectado a dois banhos de água com temperaturas controladas para analisar o comportamento reológico de amidos durante a gelatinização.

O Brookfield modelo RVDV-II+PRO é um viscosímetro rotacional comumente acoplado a um banho de circulação com controle programado de temperatura para a avaliação da viscosidade durante o aquecimento e resfriamento de soluções ou suspensões viscosas. Por ser um aparelho de custo acessível, o viscosímetro Brookfield pode ser utilizado com vantagens na caracterização reológica precisa de fluidos, contudo como padrão internacional, a referência utilizada é a determinada pelo RVA-4, visto que é um equipamento específico para análise de amidos e farinhas.

Desta forma, o objetivo deste trabalho foi comparar os equipamentos Brookfield modelo RVDV-II+PRO e Rápido Viscoanalisador modelo RVA-4 para a avaliação do perfil viscoamilográfico de diferentes amidos, usando-se as mesmas condições de análise. A comparação entre os métodos teve por objetivo permitir a comparação direta entre as medidas obtidas nos dois sistemas para facilitar a utilização dos equipamentos, principalmente em empresas, para o controle de qualidade e desenvolvimento de produtos.

\section{Material e métodos}

\section{Material}

Amidos de mandioca (Manihot esculenta), milho (Zea mays), mandioquinha-salsa (Arracacia xanthorrhiza) nativos e uma amostra de polvilho azedo foram adquiridos no comércio local, enquanto amidos ácido-modificados a $0,15 \mathrm{~mol} \mathrm{~L}^{-1}(\mathrm{HCl})$ e amido oxidado com $\mathrm{NaClO}$ a 2,0 $\%(\mathrm{v} / \mathrm{m})$ foram preparados, em laboratório, de acordo com Silva et al. (2001), empregando-se água deionizada como solvente. As amostras foram submetidas a análises sob as mesmas condições nos viscosímetros rotacionais Brookfield ${ }^{\circledR}$ modelo RVDV-II+PRO e Rápido Visco-analisador modelo 
RVA- $4^{\circledR}$ utilizando-se os softwares Rheocalc $^{\circledR}$ v.3.0 e Thermocline for Windows ${ }^{\circledR}$ para coleta e préprocessamento dos dados em cada um dos equipamentos, respectivamente.

\section{Métodos}

As medidas de viscosidade aparente foram realizadas com registros de dados comuns de temperatura $\left({ }^{\circ} \mathrm{C}\right)$, rotação $(160 \mathrm{rpm})$ e tempo (s), e dados variáveis de torque $(\%)$ no Brookfield RVDV-II+PRO (torque máximo $7.187 \mathrm{dyn}-\mathrm{cm}$, adaptador para pequenas amostras SC4-13RPY e MVS1Y Flag Impeller Spindle) e de viscosidade aparente (cP) no RVA-4.

Figura 1 - Princípio de operação de viscosímetro rotacional.

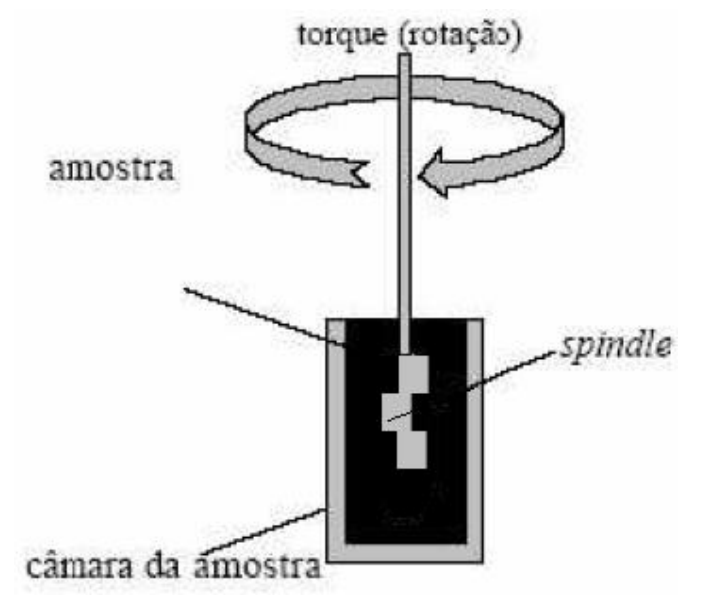

FONTE: Manual de operação. BROOKFIELD (2009).

As amostras foram suspensas em água deinizada nas concentrações de $6,67 \%(\mathrm{~m} / \mathrm{m}, \mathrm{BS})$ para todos os amidos (amidos nativos de mandioca, milho regular, mandioquinha-salsa e amido de mandioca ácido modificado - $\mathrm{HCl} 0,15 \mathrm{~mol} \mathrm{~L}^{-1}$ ), exceto para aquele oxidado com hipoclorito de sódio a 2,0 \% (v/m), cuja concentração da suspensão foi maior, de $20 \%$ (m/m, BS).

Cada análise realizada nos viscosímetros durou 40 minutos, sendo que o início partia de 50 ${ }^{\circ} \mathrm{C}$, com manutenção desta temperatura por um minuto para estabilizar, era elevada a $95{ }^{\circ} \mathrm{C}$ em 22 minutos, permanecendo nesta temperatura durante cinco minutos e em seguida, retornava a temperatura de $50{ }^{\circ} \mathrm{C}$ em 10 minutos, quando finalizava com manutenção por um minuto a esta temperatura. 
Figura 2 - Curva de viscosidade típica do RVA-4 mostrando os parâmetros comumente avaliados.

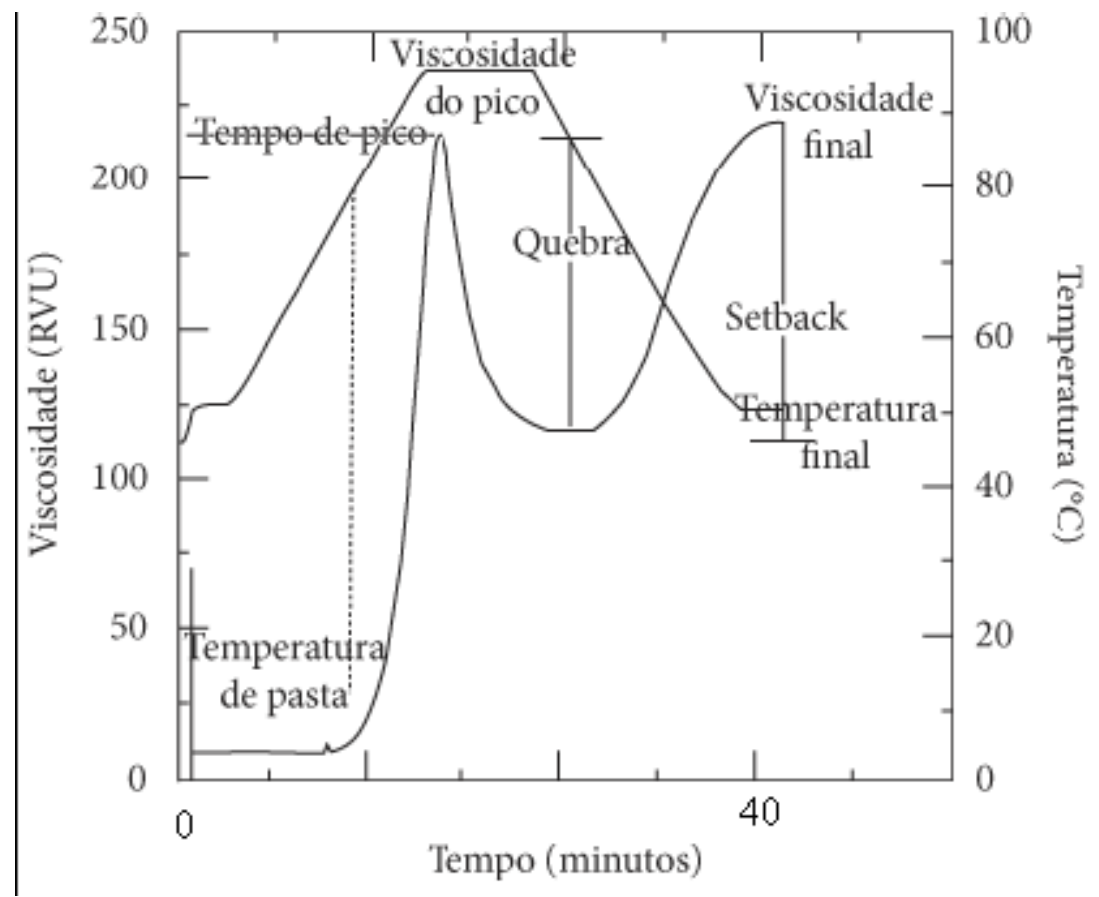

FONTE: NEWPORT SCIENTIFIC (1998).

A relação entre os equipamentos foi feita em um gráfico composto pela viscosidade aparente (cP) no eixo das coordenadas (y), e o torque (\%) no eixo das abscissas (x), partindo-se de, no mínimo, 18 pontos selecionados pelas temperaturas em comum. Este gráfico foi dividido em três curvas de tempo (s) devido ao comportamento reológico do amido apresentar três fases: (1) empastamento, (2) cozimento e (3) retrogradação. Durante a primeira etapa (fase 1), na qual a temperatura é elevada de 50 a $95{ }^{\circ} \mathrm{C}$, ocorre intensa absorção de água pelos grânulos de amido que passam por intumescimento/inchamento, gerando aumento repentino da viscosidade da suspensão, cuja temperatura e intensidade dependem do tipo de amido (origem botânica, tipo de modificação pela qual passou) assim como da presença de solutos na água usada para suspender os grânulos. A fase 2 é caracterizada pelo comportamento dos grânulos de amido gelatinizados quando estão sendo submetidos simultaneamente a agitação mecânica e cozimento, com manutenção da temperatura a $95{ }^{\circ} \mathrm{C}$. Em função de características estruturais, alguns grânulos se rompem parcial e outros totalmente, liberando polissacarídeos na água de cozimento, havendo ainda alguns que resistem a essa ruptura. Dessa forma os amidos podem ser considerados de fácil ou de difícil cozimento, com menor ou maior estabilidade ao cozimento sob agitação. A instabilidade, neste caso, é revelada pela intensidade de decréscimo da viscosidade aparente da pasta de amido, representada na Figura 2 como quebra. A fase 3 é caracterizada pela elevação da viscosidade aparente que ocorre em decorrência do resfriamento das pastas de amido, de 95 para $50{ }^{\circ} \mathrm{C}$. Há uma tendência de as cadeias 
polissacarídicas se re-associarem, com aumento na viscosidade aparente, que na Figura 2 é representado como setback.

Para padronizar os dados para estabelecimento de parâmetros que facilitassem a interconversão dos resultados em cada comportamento das pastas, neste trabalho os viscoamilogramas foram divididos nessas três fases.

\section{Resultados e discussão}

A Figura 3 mostra um exemplo da avaliação do comportamento da pasta de amido nativo analisada sob mesmas condições nos viscosímetros Brookfield RVDV-II+PRO e RVA-4.

Figura 3 - Viscoamilogramas obtidos nos viscosímetros Brookfield e RVA-4 para amidos nativos e modificados em água deionizada
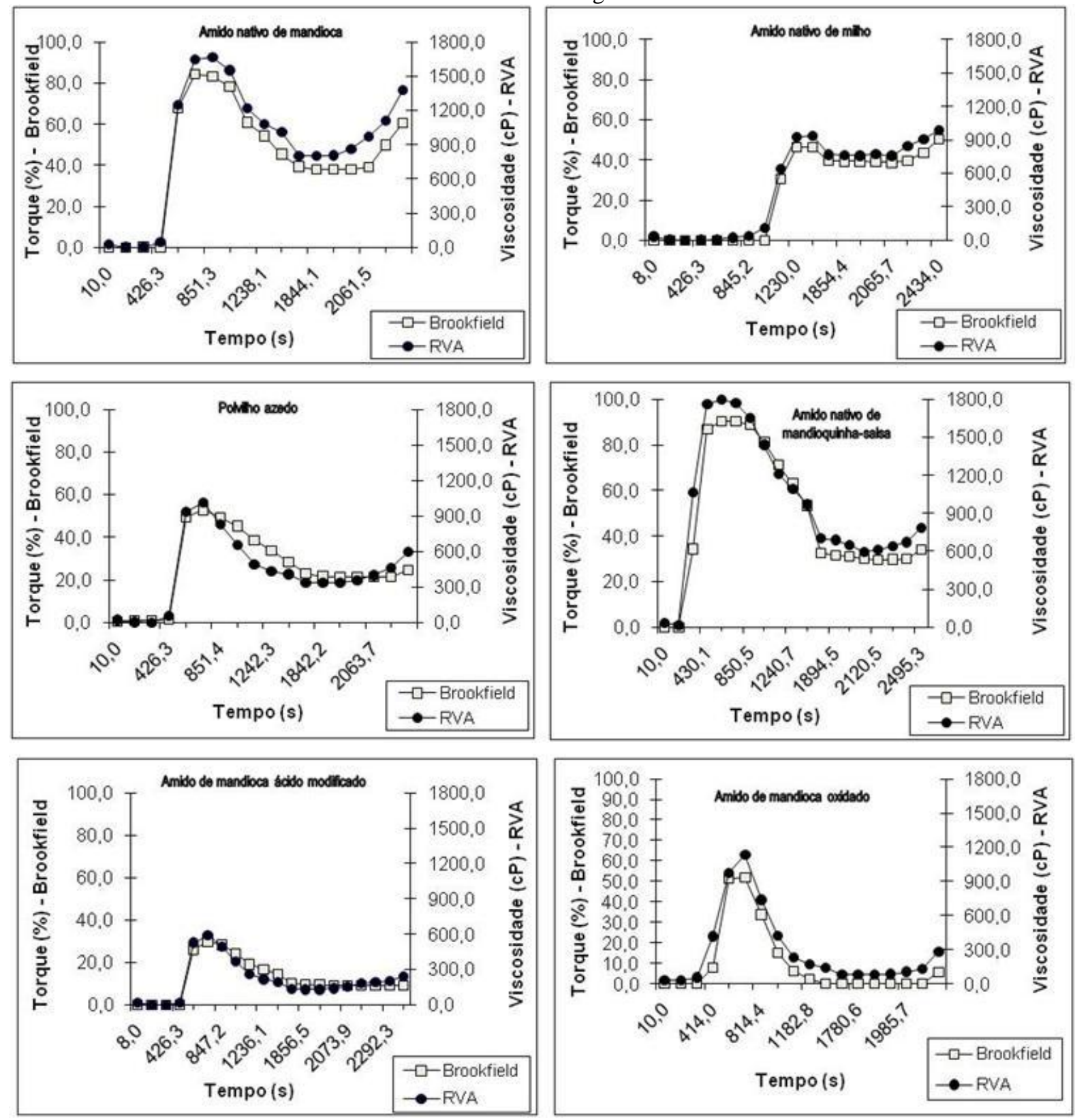
De acordo com a Figura 3, verificou-se que para as mesmas amostras, os valores de porcentagem de torque em um viscosímetro e de viscosidade aparente em outro, como esperado, estão diretamente relacionados. Para todas as amostras avaliadas foi possível obter esta semelhante relação por intermédio dos 18 pontos selecionados pelas temperaturas em comum, registradas pelos respectivos softwares.

Devido ao comportamento reológico do amido ser analisado em três fases (empastamento, cozimento e resfriamento), foram obtidas três curvas para cada amostra sendo estas as responsáveis pela interconversão dos valores de um equipamento para outro através de equações apresentadas na Tabela 1.

Tabela 1 - Equações das amostras para cada uma das fases e para os diferentes amidos

\begin{tabular}{|c|c|c|c|}
\hline Amostra & Fase & Equação polinomial & $\mathbf{R}^{2}$ \\
\hline \multirow{3}{*}{ Amido Mandioca } & $1-\mathrm{t} \leq 598,6 \mathrm{~s}$ & $y=0,0715 x^{2}+13,251 x+17,77$ & 0,9994 \\
\hline & $2-598,6 s<t \leq 2074,6 s$ & $y=0,0188 x^{2}+16,528 x+152,19$ & 0,9918 \\
\hline & $3-t>2074,6 s$ & $y=0,2469 x^{2}-3,5938 x+685,73$ & 0,9711 \\
\hline \multirow{3}{*}{ Amido Milho } & $1-t \leq 1172,6 s$ & $y=-0,0401 x^{2}+21,178 x+29,333$ & 0,9906 \\
\hline & $2-1172,6 \mathrm{~s}<\mathrm{t} \leq 2136,1 \mathrm{~s}$ & $y=2,4962 x^{2}-188,09 x+4301,7$ & 0,9944 \\
\hline & $3-\mathrm{t}>2136,1 \mathrm{~s}$ & $y=-1,4701 x^{2}+148,25 x-2753,7$ & 0,9231 \\
\hline \multirow{3}{*}{$\begin{array}{c}\text { Amido } \\
\text { Mandioquinha } \\
\text { Salsa }\end{array}$} & $1-\mathrm{t} \leq 524,8 \mathrm{~s}$ & $y=-0,1949 x^{2}+37,245 x+13,77$ & 0,9998 \\
\hline & $2-1524,8 \mathrm{~s}<\mathrm{t} \leq 2058,2 \mathrm{~s}$ & $y=0,1583 x^{2}-1,475 x+552,84$ & 0,9918 \\
\hline & $3-t>2058,2 s$ & $y=-19,135 x^{2}+1255,6 x-19783$ & 0,9944 \\
\hline \multirow{3}{*}{ Polvilho Azedo } & $1-\mathrm{t} \leq 533,0 \mathrm{~s}$ & $y=-0,0028 x^{2}+19,199 x+3,7783$ & 0,9988 \\
\hline & $2-533,0 \mathrm{~s}<\mathrm{t} \leq 2054,1$ & $y=0,6246 x^{2}-27,531 x+645,74$ & 0,9858 \\
\hline & $3-\mathrm{t}>2054,1 \mathrm{~s}$ & $y=-249,19 x^{2}+11481 x-131101$ & 0,9725 \\
\hline \multirow{3}{*}{$\begin{array}{l}\text { Amido Ácido- } \\
\text { modificado } 0,15 \\
\text { mol L }^{-1}\end{array}$} & $1-\mathrm{t} \leq 541,2 \mathrm{~s}$ & $y=-0,0431 x^{2}+20,971 x+9$ & 0,9995 \\
\hline & $2-541,2 s<t \leq 2156,6 s$ & $y=0,592 x^{2}-3,2419 x+107,77$ & 0,9994 \\
\hline & $3-t>2156,6 s$ & $y=1050 x^{2}-18625 x+82780$ & 0,9656 \\
\hline \multirow{3}{*}{$\begin{array}{c}\text { Amido Oxidado } \\
2,0 \%\end{array}$} & $1-\mathrm{t} \leq 500,2 \mathrm{~s}$ & $y=-0,6435 x^{2}+52,756 x+46,31$ & 0,9878 \\
\hline & $2-500,2 \mathrm{~s}<\mathrm{t} \leq 2127,9 \mathrm{~s}$ & $y=-0,1286 x^{2}+23,309 x+98,61$ & 0,9924 \\
\hline & $3-t>2127,9 s$ & $y=31,879 x+107,67$ & 0,9557 \\
\hline
\end{tabular}

É importante destacar que na Tabela 1 os valores que definem o início e término das três fases são distintos e isso se deve ao fato de que amidos de diferentes origens botânicas têm comportamentos viscoamilográficos (reológicos) distintos. O amido de mandioquinha-salsa, por exemplo, apresenta a mais baixa temperatura de empastamento enquanto o amido de milho regular tem temperatura de empastamento mais elevada. Da mesma forma, a modificação química dos amidos altera os parâmetros dos viscoamilogramas, alterando a temperatura de empastamento (fase 1), a estabilidade ao cozimento sob agitação (fase 2) e a tendência à retrogradação (fase 3).

A possibilidade de utilização de um sistema alternativo aos viscoamilógrafos convencionais é interessante para as empresas que podem economizar na aquisição desses equipamentos e poderão obter resultados comparáveis e relevantes 


\section{Conclusões}

Foram obtidos valores comparáveis entre os dois viscosímetros testados o que possibilita a adoção de qualquer um deles para a obtenção de viscoamilogramas de simples interpretação e que podem ser utilizados pelas empresas na emissão de laudos de qualidade de amidos e outros produtos amiláceos.

Através das relações obtidas nos gráficos, verificou-se que os valores de $\mathrm{R}^{2}$ foram próximos de um, variando de 0,9231 a 0,9995, o que comprovou a proximidade e possibilidade de interconversão de valores entre os dois equipamentos por intermédio das equações de comportamento das amostras em suspensão aquosa.

\section{Referências}

BILIADERIS, C. G. The structure and interactions of starch with food. Canadian Journal of Phisiology and Pharmacology, v. 69, p. 60-78, 1991.D.O.I: 10.1139/y91-011

BROOKFIELD. Manual de operação viscosímetro modelo RVDV-II+PRO. Disponível em <http://www.brookfieldengineering.com>, acesso em 13/01/2010.

CEREDA, M. P. Propriedades gerais do amido. São Paulo, Fundação Cargill, 221 p. (Série: Culturas de tuberosas amiláceas Latino-americanas, v. 1), 2001.

CEREDA, M. P.; VILPOUX, O. F. Tecnologia, usos e potencialidades de tuberosas amiláceas Latino Americanas. São Paulo: Fundação Cargill, 711p. (Culturas de Tuberosas Amiláceas Latino Americanas, v.3), 2003.

CEREDA, M. P.; WOSIACKI, G. Characterization of pinhão starch. Part I. extraction and properties of the starch granules. Starch/Stärke, Weinheim, v. 37, p. 224-227, 1985.

DEFFENBAUGH, L. B; WALKER, C. E. Comparasion of starch pasting properties in the Brabender Viscoamylograph and the Rapid Visco-Analyzer. Cereal Chemistry, v. 66, n. 6, p. 493-499, 1989.

NEWPORT SCIENTIFIC. Manual de operação RVA-4. 1998.

ROSS, A. S.; WALKER, C. E.; BOOTH, R. I.; ORTH, R. A.; WRIGLEY, C. W. The Rapid Visco Analyser: A new technique for the estimation of sprout damage. Cereal Foods World, v. 32, p. 827-829, 1987.

SILVA, R. M; FERREIRA, G. F; SHIRAI, M. A; HASS, A; SCHERER, M. L; FRANCO, C. M. L; DEMIATE I. M. Características físico-químicas de amidos modificados com permanganato de potássio/ácido lático e hipoclorito de sódio/ácido lático. Ciência e tecnologia de Alimentos, v. 28, n. 01, p. 66-77, 2008. D.O.I: :10.1590/S010120612008000100011

SILVEIRA, C. A. Uso do viscosímetro Brookfield em determinações reológicas. Polímeros: Ciência e Tecnologia. p. 41-43, nov/dez, 1991.

STEFFE, J.F.; CASTELL-PEREZ, M.E.; ROSE, K.J.; ZABIK, M.E. Rapid testing method for characterizing the rheological behavior of gelatinizing corn starch slurries. Cereal Chemistry, v.66, n.1, p.65-68, 1989.

THOMAS, D. J.; ATWELL, W. A. Practical Guide for Food Industry In: Starches. Minnesota: Eagan Press, 1999. 30p.

VILLETTI, M. A. Determinação do coeficiente de viscosidade pelo viscosímetro de Ostwald, 2003. Disponível em <http://w3.ufsm.br/juca/viscosidade.pdf>, acesso em 13/01/2010. 
VILPOUX, O. Amidos adaptados ao uso nas indústrias de alimentos. Fax/Jornal CERAT/UNESP, Botucatu, n. 70, p. 1-2, 1998.

WHISTlER, R. L.; BEMILlER, J. N. Carbohydrate Chemistry for Food Scientists. San Diego: Academic Press, 1997. $214 \mathrm{p}$.

\begin{abstract}
Rotary viscometers are used to measure the viscosity of a variety of products including many applications in the food industry, including analyses of suspensions and solutions of polysaccharides such a starch. In this work the comparison between two devices, the Brookfield RVDV-II + PRO and the Rapid Visco-Analyser RVA-4 was made, using the same conditions of analysis and aimed specifically to allow direct comparison between measurements obtained in both systems to facilitate their use by the industry. Starch pastes were produced in deionized water and subjected to analysis under the same conditions in the different viscometers. The results were obtained from the relationship that was done on graph composed of apparent viscosity (cP) and torque (\%), starting from at least 18 points selected at the same temperatures. What has been noted by the analyses conducted with the native starches from cassava, corn and Peruvian carrot, acid modified cassava starch $\left(0.15 \mathrm{~mol} \mathrm{L^{-1 }} \mathrm{HCl}\right), \mathrm{NaClO}$ oxidized $(2.0 \%$ of active chlorine) cassava starch and cassava sour starch, was that for the same samples, the values of torque in a viscometer and apparent viscosity in the other were directly related, yielding $R^{2}$ values close to 1 , confirming the proximity and possibility of interconversion between the values of the two equipments by using the equations for each behavior.
\end{abstract}

Key-words: Viscometer; RVA-4; apparent viscosity; starch.

Submetido em 16 dez. 2010; Revisão em 15 mai. 2011; Aceito para publicação em 30 jun.2011. 